\title{
Relationship Between Permeation and Deformation for Porous Membranes
}

\author{
Masoud Aghajani, ${ }^{1}$ Sajjad H. Maruf, ${ }^{1}$ Mengyuan Wang, ${ }^{2}$ Joseph Yoshimura, ${ }^{1}$ Gabriel \\ Pichorim, ${ }^{3}$ Alan Greenberg, ${ }^{1}$ and Yifu Ding ${ }^{1,2, *}$ \\ ${ }^{1}$ Membrane Science, Engineering and Technology Center \\ Department of Mechanical Engineering \\ University of Colorado Boulder, Boulder, CO 80309-0427, USA \\ ${ }^{2}$ Materials Science and Engineering Program, \\ University of Colorado, Boulder, CO 80309-0596, USA \\ ${ }^{3}$ UFRN - Federal University of Rio Grande do Norte, \\ Senador Salgado Filho Avenue, 3000 - Lagoa Nova, \\ Natal - RN, Brazil, 59064-741
}

\begin{abstract}
Most polymeric membranes are engineered to have high porosity in order to reduce transport resistance of the permeate(s). However, since most polymers are relatively compliant and exhibit time-dependent behavior, such membranes are prone to deformation under mechanical loading that can occur during different stages of manufacturing as well as during separation. Therefore, it is of critical importance to understand the influence of mechanical deformation on the permeability of the membranes. However, an appropriate relationship has not been established due to the lack of methods that can precisely control membrane deformation and the significant variability of membrane properties. Here, we report the systematic and quantitative examination of the permeability-deformation relationship for microfiltration (MF) and ultrafiltration (UF) membranes with different pore structure and chemistry. Polymer membranes were deformed to different levels of compressive strain using nanoimprint lithography with systematically different processing conditions. Furthermore, permeation measurements were carried out on each membrane sample before and after the compressive deformation to minimize membrane variability concerns. The experimental results reveal that the permeability of the MF and UF membranes decreases with the increase of compressive strain such that the dependency approaches the behavior of an open-cell foam. The derived relationship is simple but useful despite key differences between open-cell foams and porous membranes including pore structure asymmetry and the much smaller pore size of the latter.
\end{abstract}

${ }^{*}$ Corresponding author: Department of Mechanical Engineering, 427 UCB, University of Colorado, 1111 Eng. Drive, Boulder, CO 80309-0427; Tel.: +1 303492 2036; E-mail address: Yifu.Ding@ Colorado.Edu; Fax: +1 303-492-3498. 


\section{Introduction}

Polymeric membranes are widely used in separation processes with applications ranging from particulate removal to desalination [1]. Membranes are generally engineered to have high porosity in order to minimize permeate flow resistance. However, high porosity in combination with the relatively low stiffness of the polymers has significant implications regarding the mechanical robustness of these membranes. During manufacture, flat-sheet membranes are often folded via pleating (into cartridges) or rolled up and then compressed by spacers (into modules) [2], either of which can cause severe local deformation of the membranes.

Perhaps the most well-known effect related to membrane deformation is the compaction, i.e. compressive creep, that occurs during active separation [3-8]. In pressure-driven separation processes such as ultrafiltration (UF), nanofiltration (NF), and most significantly high-pressure reverse osmosis $(\mathrm{RO})$, a decrease in permeate flux with time at constant operating pressure is commonly observed with polymeric membranes, even in the absence of fouling. During RO, Mashiko et al showed that cellulose acetate butylate membranes experienced a $30-40 \%$ instantaneous thickness reduction upon pressurization, followed by additional thickness reduction over time but at a slower rate [9]. Using ultrasonic time-domain reflectometry (UTDR), Peterson et al determined the time-dependence of membrane compression during $\mathrm{RO}$, and determined that both total compressive strain as well as the irrecoverable strain (after unloading) increased with an increase of filtration pressure [10]. Huisman et al showed that the resistance of MF and UF membranes increased with an increase of the transmembrane pressure, which was attributed to the deformation of the membranes [11]. Persson et al [12] compared the effect of pre-compression on the subsequent deformation and permeance of UF membranes. Their results showed that polysulfone (PS) membranes displayed a larger degree of mechanical deformation than cellulose 
acetate (CA) membranes under similar pressure. Correspondingly, greater flux decline was observed for the PS membranes. These differences were attributed to the higher porosity and thus the greater degree of plastic deformation of the PS membranes. Although most of these reports associated the flux decline with the morphological changes of the porous membrane due to pressurization, the direct quantitative correlations remain unclear.

One method to study compaction is mechanical testing $[5,12,13]$. Using this approach, a compressive stress is applied onto the membrane with or without subsequent use in filtration under realistic operating conditions. Deanin et al. [13] used a water bath and a high pressure of 10.3 MPa (1500 psi) applied via a stainless steel rod to compare the viscoelastic creep behavior of CA membranes with and without fillers. Mashiko et al. [9] used a hydraulic machine with two cylindrical rods to apply force (stress) and study the thickness change of the membrane over the period of stress application.

In contrast to the negative implications of membrane deformation, the plasticity associated with high degrees of porosity can be utilized to create engineered roughness, i.e. periodic patterns, on the surface of commercial membranes $[14,15]$. Specifically, using thermal embossing through nanoimprint lithography (NIL), the pattern features on a suitable Si mold can be replicated atop the surface of the membranes via porosity-induced plasticity under controlled conditions. The presence of the surface patterns has been shown to enhance the antifouling characteristics of modified UF [16, 17] and thin film composite (TFC) [18, 19] membranes. However, during the pattern replication process, the entire membrane is compressed such that an increase of intrinsic membrane resistance can result. Thus, to apply this patterning technology to a broad range of membranes, it is of critical importance to quantify the effect of membrane deformation during NIL on the subsequent membrane resistance [20]. 
The above literature review suggests that mechanical loading, i.e. pressurization, whether during manufacturing or operation, alters the membrane structure and consequently the resistance to permeate flow. However, an evidence-based mathematical relationship between membrane deformation and resistance is currently lacking. This is not surprising given that most of the available literature data is for RO membranes, where the multilayer composite structure makes it challenging to isolate the mechanical (strain) and physical (resistance) responses of the porous layer. In this study, we quantify the association between membrane deformation and permeability for several UF and MF membranes as a function of pore structure and polymer chemistry. The critical finding of this work is that membrane permeability decreases with an increase of compressive strain such that the dependency approaches the behavior of an open-cell foam.

\section{Experimental}

\subsection{Materials}

Four different membranes supplied by MilliporeSigma were utilized for this study: a polyethersulfone (PES) UF membrane, a polysulfone (PS) UF membrane and two Durapore ${ }^{\mathrm{TM}}$ polyvinylidene fluoride (PVDF) MF membranes. Of these, only the PS membrane had a nonwoven support layer. As indicated in Table 1, relevant physical and morphological properties of the four membranes were evaluated; the PES membrane, hereafter identified as PES-A14, was subjected to a more detailed characterization as described below. All of the membranes were received as flat-sheets, stored in an ambient dry environment and subsequently used without further treatment unless otherwise specified. Chemicals utilized in this study included acetone and isopropanol both from Fisher Scientific (Certified ACS grade with purity above 99.5\%). Flux measurements were conducted using deionized (DI) ultrapure water with a resistivity of $10 \mathrm{M} \Omega$ $\mathrm{cm}$. 
Table 1 Characteristics of the membranes used for this study.

\begin{tabular}{cccccc} 
Membrane & Polymer & Pore Size $(\mathrm{nm})$ & $T_{g}\left({ }^{\circ} \mathrm{C}\right)$ & $T_{m}\left({ }^{\circ} \mathrm{C}\right)$ & $\begin{array}{c}\text { Fiber } \\
\text { Support }\end{array}$ \\
\hline PES-A14 & Polyethersulfone & $20-50^{\mathrm{a}}$ & $220^{\mathrm{d}}$ & - & No \\
PS & $\begin{array}{c}\text { Polysulfone } \\
\text { Purapore }\end{array}$ & $30 \mathrm{kD}^{\mathrm{b}}$ & $200-210^{\mathrm{d}}$ & - & Yes \\
DVPP & $\begin{array}{c}\text { Polyvinylidene } \\
\text { Fluoride }\end{array}$ & $650^{\mathrm{c}}$ & - & 182.2 & No \\
$\begin{array}{c}\text { Durapore } \\
\text { VVPP }\end{array}$ & $\begin{array}{c}\text { Polyvinylidene } \\
\text { Fluoride }\end{array}$ & $100^{\mathrm{c}}$ & - & 165.1 & No \\
\hline
\end{tabular}

${ }^{\mathrm{a}}$ Measured by top-down SEM image of the membrane surface. ${ }^{\mathrm{b}}$ Pore size is too small to visualize due to FE-SEM resolution and the masking by gold-layer. The molecular weight cut off (MWCO) is 30k Dalton according to manufacturer specification. ${ }^{\mathrm{c}}$ Nominal pore size of the membrane from the specification sheet provided by manufacturer. ${ }^{\mathrm{d}}$ Based on the onset temperature of the DMA-test modulus decrease.

\subsection{Controlled membrane deformation via NIL}

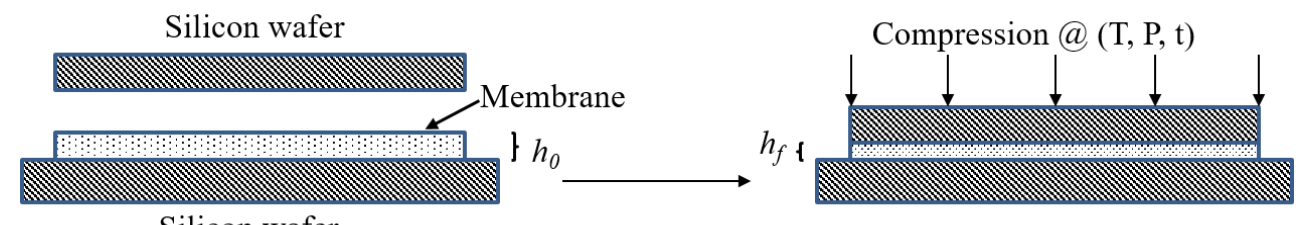

Figure 1. Schematic illustration of membrane compression using NIL process. By applying uniform pressure $P$ at temperature $T$ for $t$ seconds, membrane thickness is reduced from $h_{0}$ to $h_{\mathrm{f}}$.

Nanoimprint lithography (NIL, Eitre 3, Obducat, Sweden) was used to uniaxially compress the membranes under prescribed conditions of temperature, pressure and duration. As illustrated in Fig. 1, the circular membrane samples (diameter: $\sim 5 \mathrm{~cm}$ ) were placed between two silicon (Si) wafers and then compressed. Both the air-cushion technology used in the Eitre 3 and the smooth surface of Si wafer allow uniform compression across the membrane surface (1\% variation over a 3-inch wafer diameter), and has been utilized to reliably generate controlled nm-scale deformation onto polymer surfaces [21] and even particles [22, 23]. For all of the membrane samples, the duration of the NIL-generated compression was held constant at $180 \mathrm{~s}$, while the pressure and 
temperature were systematically varied to achieve different levels of compressive strain. After the system was cooled to ambient temperature, the compressed membranes were separated from the upper and lower silicon wafers (platens). DI water flux for each sample was determined before and after compression, which minimizes the influence of intrinsic sample-to-sample variability.

\subsection{Membrane characterization}

The glass transition temperature $\left(T_{g}\right)$ of the PES UF membrane was determined using dynamic mechanical analysis (DMA, TA Instruments Q800). The membranes were sectioned into $15 \mathrm{~mm} \times 6.5 \mathrm{~mm}$ rectangular strips for all DMA measurements, which were carried out under tension at a dynamic loading frequency of $10 \mathrm{~Hz}$ and a strain amplitude of $0.1 \%$ while the temperature was increased from 30 to $250{ }^{\circ} \mathrm{C}$ at a controlled heating rate of $5{ }^{\circ} \mathrm{C} / \mathrm{min}$. The $T_{g}$ value was defined as the temperature corresponding to the maximum of the tan $\delta$ damping peak. For the two semi-crystalline PVDF membranes, the melting temperature $\left(T_{m}\right)$ as well as the crystallinity of the PVDF were determined using differential scanning calorimetry (DSC 204 F1 Phoenix, NETZSCH) at a scanning rate of $2{ }^{\circ} \mathrm{C} / \mathrm{min}$.

The mechanical properties of an amorphous (PES-A14) and a semi-crystalline membrane (PVDF-VVPP) were characterized via a uniaxial tensile test using the DMA apparatus. Specifically, isothermal stress-strain curves for the membranes were determined at a constant strain rate. For the PES-A14, measurements were carried out at 40, 80, 120 and $160{ }^{\circ} \mathrm{C}$ while for PVDF, the temperatures utilized were $25,50,75$ and $100{ }^{\circ} \mathrm{C}$. At each temperature, tests were conducted at three strain rates: $0.0001,0.001$, and $0.01 \mathrm{~s}^{-1}$. A 10-min waiting period was adopted before each measurement to ensure that the temperature throughout the membrane sample was uniform and reached the designated value. Three different specimens were tested at each condition 
(strain rate and temperature) so that a mean and standard deviation for each condition could be obtained.

The thickness $(l)$ of the membranes was measured using a micrometer (Chicago Dial Indicator). For each sample, ten randomly selected locations were sampled and the mean and standard deviation were calculated. Porosity for the PES-A14 compressed membranes was determined by measuring the difference in mass between the wet and dry samples. To minimize the effect of incomplete pore-wetting, a vacuum-assisted liquid-wetting method was applied using isopropanol (IPA) as the wetting liquid. The porosity $(\varphi)$ of the membrane is defined as,

$$
\varphi=\frac{w_{1}-w_{2}}{\rho A l}
$$

where $w_{1}$ and $w_{2}$ correspond to the mass of the wet and dry membrane, respectively, $\rho$ is the density of the wetting liquid, and $A$ is the membrane sample area. For each sample, three replicate measurements were carried out and the mean and standard deviations calculated.

Field-emission scanning electron microscopy (FESEM, Supra 60, Zeiss) was used to image the cross-section as well as the selective surface of the membranes. The samples for the crosssectional SEM imaging were prepared by liquid nitrogen fracture and subsequent drying in a vacuum oven. A 4-5 nm gold layer was coated on each sample prior to SEM imaging to minimize sample charging. Analysis of surface SEM images was carried out using ImageJ. A brightness threshold of 71 is used to calculate surface -pore coverage.

A dead-end filtration cell (XX4504700, MilliporeSigma) with an active surface area of 9.6 $\mathrm{cm}^{2}$ and pressurized with nitrogen was used for all of the DI water permeation measurements. The permeate flow rate was measured with an automated electronic balance (PI-225DA, Denver Instrument) at 2-min intervals. For a given membrane sample, DI water flux was determined at 
$207 \mathrm{kPa}$. At each pressure, the steady-state flux was defined as the flux with less than $2 \%$ variation

over a 10-min interval. Before each filtration experiment, the membrane was soaked in an isopropanol/DI water solution ( $20: 80$ mixing ratio) for $30 \mathrm{~min}$ to pre-wet the membrane; all experiments were conducted at room temperature.

Relevant physical and morphological properties of the four membranes were obtained and are summarized in Table 1. Because of its relatively uniform structure, absence of macrovoids, and lack of a backing layer, the PES-A14 membrane was utilized as the primary reference material and thus subjected to a more detailed characterization protocol than the other three membranes.

\section{Results and discussion}

Figure 2a shows the storage modulus as a function of temperature from DMA measurements for both PES-A14 (amorphous) and PVDF-VVPP (semi-crystalline). PES-A14 displayed behavior typical of an amorphous polymer, i.e. the modulus shows weak temperature dependence until reaching the glass transition region of the PES where the modulus then decreases significantly. The $T_{g}$ of the PES-A14 was estimated as $\sim 220{ }^{\circ} \mathrm{C}$ based upon the onset of the modulus decrease or $\sim 236{ }^{\circ} \mathrm{C}$ based on the maximum of the tan $\delta$ peak (Fig. S1), each of which are in good agreement with reported values for bulk PES [24]. In contrast, the storage modulus of PVDF-VVPP exhibited a much stronger temperature dependence, showing a one-order of magnitude reduction from 25 to $140{ }^{\circ} \mathrm{C}$, followed by a rapid decrease upon further heating. From DSC measurements (Fig. S2), the $T_{m}$ of the PVDF membranes were estimated to be $\sim 165.1^{\circ} \mathrm{C}$ and $182.2^{\circ} \mathrm{C}$, respectively, for VVPP and DVPP (Table 1). The melting enthalpies $\left(\Delta H_{m}\right)$ for VVPP and DVPP were also obtained as $54.7 \mathrm{~J} / \mathrm{g}$ and $62.8 \mathrm{~J} / \mathrm{g}$, respectively. Using $\Delta H_{0}=109.0 \mathrm{~J} / \mathrm{g}$ as the reference value for $100 \%$ crystalline PVDF [25], the crystallinity of the PVDF membrane was 
estimated from the ratio of $\Delta H_{\mathrm{m}} / \Delta H_{0}$ as $50.2 \%$ and $57.6 \%$ for VVPP and DVPP, respectively. Between $T_{g}$ and $T_{m}$ (Fig. 1a), the amorphous PVDF regions become rubbery with a continuous reduction of the storage modulus. Above $T_{m}$, the material becomes viscous with a more pronounced decrease in the stiffness.

(a)

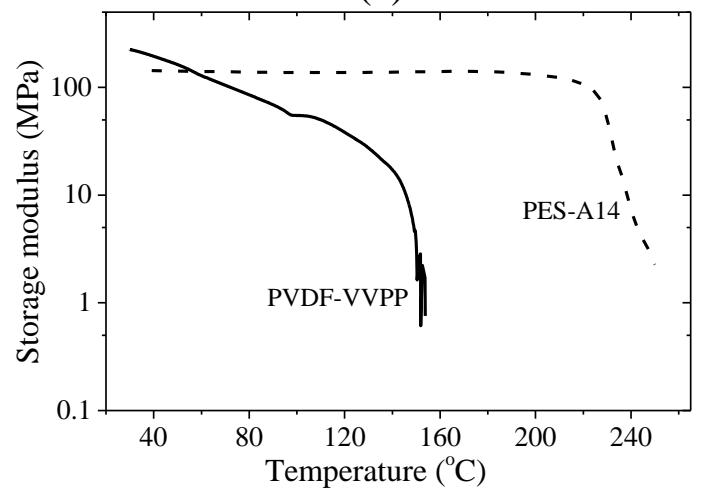

(c)

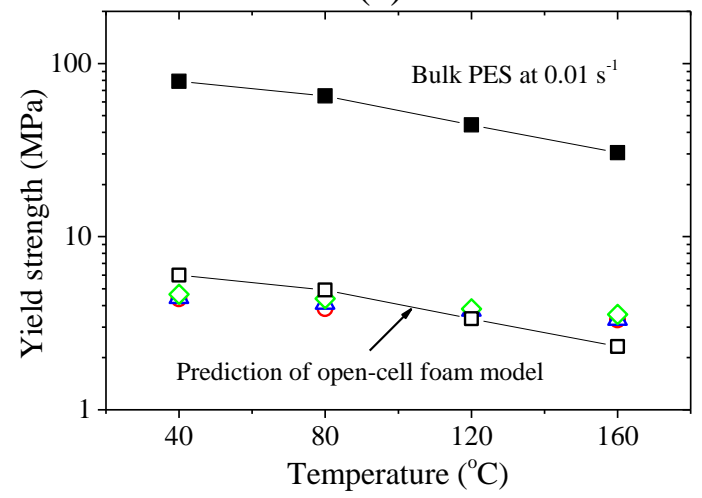

(b)

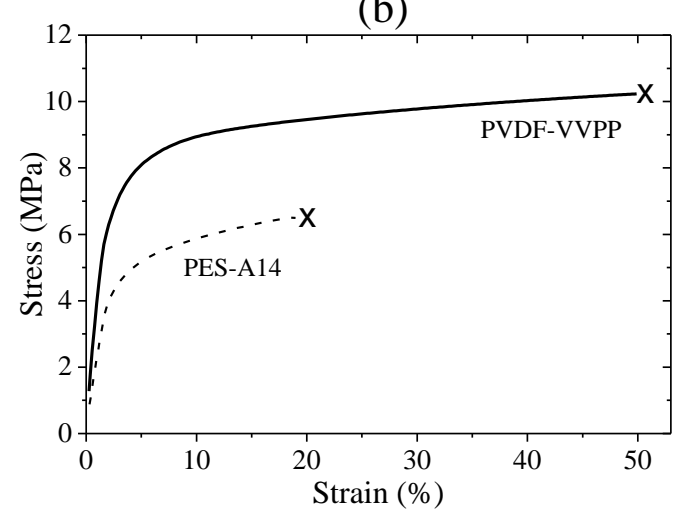

(d)

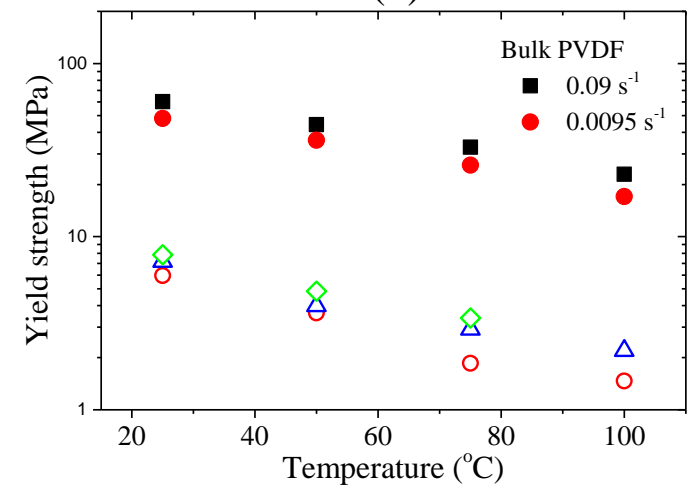

Figure 2. (a) Storage modulus as a function of temperature from DMA measurements for PESA14 and PVDF-VVPP. (b) Stress-strain curves for PES-A14 at $40{ }^{\circ} \mathrm{C}$ and PVDF-VVPP at $25{ }^{\circ} \mathrm{C}$ measured under uniaxial stretching at $0.01 \mathrm{~s}^{-1}$. The symbol " $\times$ " in (b) marks the failure point of the membranes. Yield strength of (c) PES-A14 and (d) PVDF-VVPP, determined using a 2\% offset from the stress-strain curves as a function of temperature for different strain rates (different symbols represent different strain rates: diamond: $0.01 \mathrm{~s}^{-1}$, triangle: $0.001 \mathrm{~s}^{-1}$, and circle: $0.0001 \mathrm{~s}^{-1}$ ). Also plotted in (c) and (d) are results for bulk PES from [26] and bulk PVDF measurements from [25]. In addition, (c) provides the predicted yield strength (square symbols) for a porous-PES membrane based upon an open-cell foam model [27]. 
When the PES-A14 and PVDF-VVPP membranes were compressed during NIL processing at the viscous state of the polymers, i.e. $T>T_{g}$ for PES and $T>T_{m}$ for PVDF, detrimental porecollapse occurred [14]. Therefore, all of the NIL-compression temperatures used were below $T_{g}$ of PES or $T_{m}$ of PVDF. Subsequently, tensile-tests were conducted for each membrane at different rates within the temperature range relevant for NIL processing. Figure $2 b$ shows representative stress-strain curves at a strain rate of $0.01 \mathrm{~s}^{-1}$ for the PES-A14 at $40{ }^{\circ} \mathrm{C}$ and PVDF-VVPP at $25^{\circ} \mathrm{C}$. Each membrane exhibited the behavior expected for a porous material: a linear elastic response at low strains $(2-3 \%)$, an extended plateau region (20 - 50\%) after apparent yielding and finally brittle failure of the sample (as marked in Fig. 2b). Note that the behavior of a porous material under NIL compression is similar to that of the tensile test through the elastic and stress plateau regions [27]. Thus, the stiffness and yield strength of the membranes under compression can be estimated from the tensile test results. The major difference between the membrane behavior in compression and tension is observed in the large-strain response where the membranes fracture under tension but densify under compression, which is related to the collapse/contact of the cell walls that prevents additional plastic deformation.

From the elastic region of the stress-strain curves (Fig. 2b), values of Young's modulus of PES-A14 and PVDF-VVPP were estimated to be $\sim 173 \mathrm{MPa}$ and $232 \mathrm{MPa}$, respectively, which agree reasonably well with the values from DMA measurements (Fig. 2a). In addition, the yield strength of each membrane, $\sigma^{*}$, can be estimated from the tensile test results using a $2 \%$ off-set [28]. The values of $\sigma^{*}$ for the PES-A14 and PVDF-VVPP membranes obtained at different temperatures and strain rates are presented in Fig. $2 \mathrm{c}$ and $2 \mathrm{~d}$, respectively. The bulk yield strength, $\sigma_{\text {bulk }}$, of PES from [26] and PVDF from [25] are also included in the figures for comparison. At all strain rates and temperatures, each membrane showed significantly lower values of $\sigma^{*}$ in 
comparison to $\sigma_{b u l k}$ due to membrane porosity. Most importantly, membrane yield strength can be reasonably well described by a relationship developed for open-cell foams [27],

$$
\frac{\sigma^{*}}{\sigma_{\text {bulk }}}=C\left(\frac{\rho^{*}}{\rho_{\text {bulk }}}\right)^{3 / 2}
$$

where $\rho^{*}$ and $\rho_{\text {bulk }}$ correspond to the density of the membrane and the bulk polymer, respectively, and $C$ is a constant with a value between 0.2 and 0.3 . Using the experimentally determined value for porosity of $59.9 \pm 4.3 \%$ and literature values for $\sigma_{b u l k}$ with $C=0.3$ (providing the best matching between the prediction and experimental data), predicted values of $\sigma^{*}$ for the PES-A14 at $0.01 \mathrm{~s}^{-1}$ were calculated and plotted in Fig. 2c. Comparison indicates that the predicted and experimental values are in reasonable agreement.

Moreover, values of $\sigma^{*}$ for both PES-A14 and PVDF-VVPP showed little dependence on strain rate within the range analyzed (relevant for NIL processing). This behavior is likely due to the unique mechanism by which viscoelastic porous polymers yield. For such membrane materials, plastic deformation is a result of structural instability (i.e. buckling) of the cell (pore) walls and simultaneous localized yielding of the polymer within the cell walls. Under mechanical loading, the cell walls can rotate/twist and buckle, and these occur at a much faster rate than the strain rates employed in the tensile test. The localized yielding of PES or PVDF that accompanies cell wall buckling occurs due to the presence of pore-induced stress concentrations. It is most likely that localized yielding is the rate-limiting step for the onset of macroscopic plastic deformation [27]. The $\sigma^{*}$ values for PVDF-VVPP show a somewhat stronger rate dependence than that of the PES because the amorphous regions of the PVDF are in the rubbery state at the testing temperatures utilized. 
The results presented in Fig. 2 demonstrate that the membranes, either amorphous or semicrystalline, can plastically deform under modest stress levels (a few MPa) at temperatures below $T_{g}$ (PES) or $T_{m}(\mathrm{PVDF})$. By changing the temperature and pressure combinations used in the NIL processing, different degrees of membrane compression can be obtained. Figure 3a shows the percent compressive strain $(\varepsilon)$ for PES-A14 obtained at different imprinting temperatures at a pressure of $4 \mathrm{MPa}$ applied for $3 \mathrm{~min}$, with

$$
\varepsilon=\left(h_{f}-h_{0}\right) / h_{0}
$$

where $h_{f}$ and $h_{0}$ are the thickness of the compressed and virgin membranes (Fig. 1), respectively. The results from Fig. 3a indicate a clear trend of increasing compressive strain with increasing imprinting temperature. Since the membrane deforms as a function of processing time at constant pressure, $\varepsilon$ represents the strain due to compressive creep. The magnitude of the compressive creep strain increases with increasing temperature due to the decrease in yield strength (Fig. 2c).

Figure $3 b$ shows that membrane porosity decreases with increasing imprinting temperature. At these high strain levels, the Poisson's ratio ( $v)$ of the membranes can be assumed to be zero, i.e. no lateral expansion during compression. Accordingly, the porosity of the membrane undergoing compression to a strain level $\varepsilon$ can be estimated (see supplementary material for a detailed derivation) from,

$$
\varphi_{\varepsilon}=\frac{\varepsilon+\varphi_{0}}{(1+\varepsilon)}
$$

where $\varphi_{0}$ represents the porosity of the virgin membrane. As plotted in Fig. 3b, the estimated porosity (empty symbols) agrees well with the experimental values (solid symbols). In addition, the results from Fig. 3c show that the DI water flux of the compressed membranes decreases monotonically with the increase of compressive strain. 


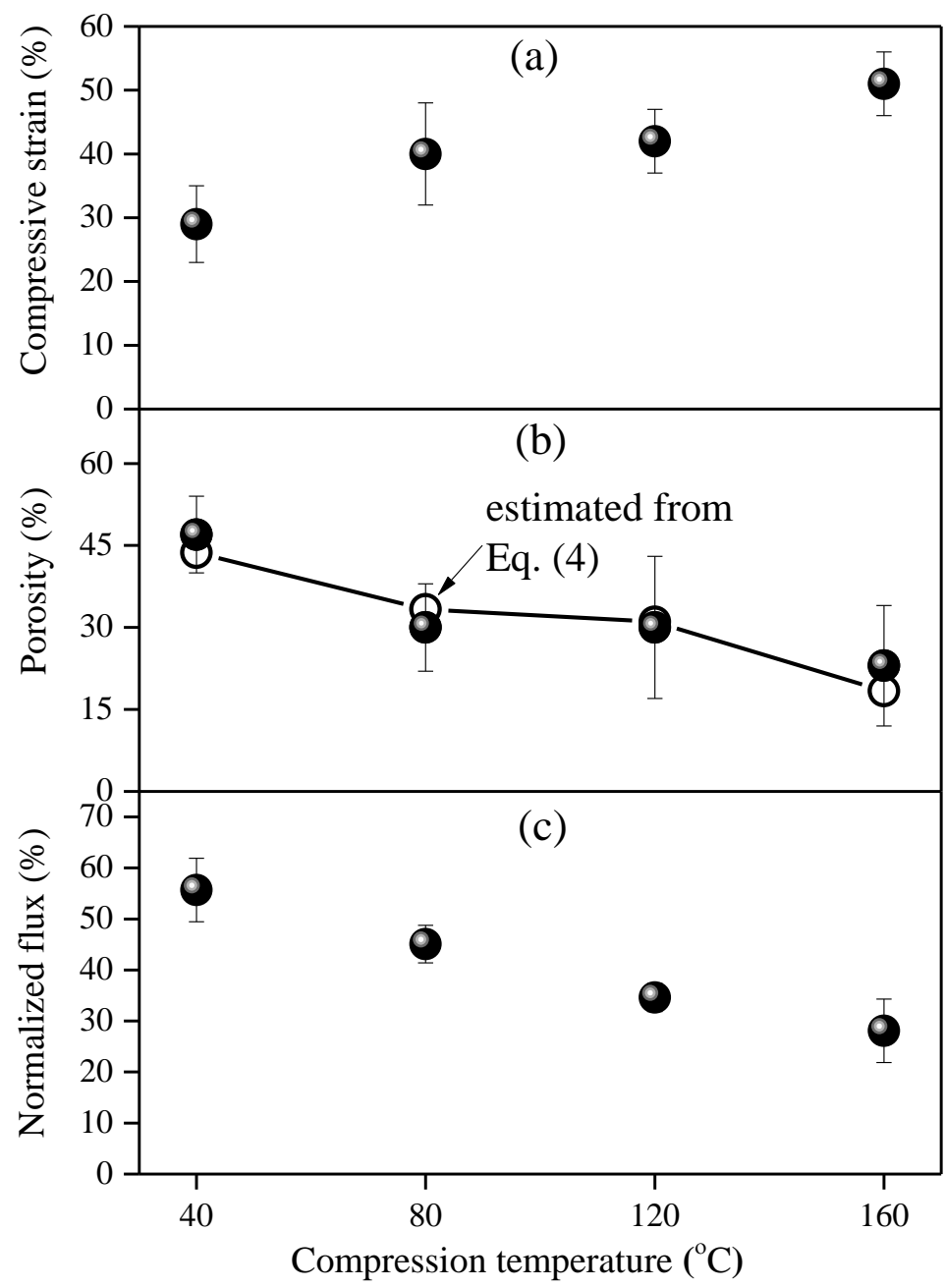

Figure 3. Influence of NIL compression temperature on the (a) compressive strain, (b) overall porosity, and (c) normalized DI water flux of the PES-A14 membranes. All membranes were compressed at $4 \mathrm{MPa}$ for $3 \mathrm{~min}$. The solid symbols represent mean values and the error bars indicate the corresponding standard deviations $(\mathrm{N}=3)$. The empty symbols (connected by solid lines) in (b) represent porosity estimated from compressive strain values using Eq. (4).

Figure 4 shows cross-sectional and top-surface micrographs of the PES-A14 membranes before and after compression at $180{ }^{\circ} \mathrm{C}$ under $4 \mathrm{MPa}$ for $3 \mathrm{~min}$. The virgin membrane evidenced an asymmetric structure with a thin selective layer atop a much thicker support layer. The pore size of the selective layer was relatively small $(<1 \mu \mathrm{m})$ and the larger pores $(5-10 \mu \mathrm{m})$ that the support layer increased gradually in size from top to bottom (Fig. 4a). Plastic buckling of the pore 
walls during compression caused significant reduction in the thickness of the membrane such that the corresponding plastic compressive strain was measured at $\sim 42 \%$ (Fig. 4b). Most importantly, the effects of compression were primarily manifested in the reduction in pore size in the support layer (Fig. 4a and 4b, insets), where the 5-10 $\mu \mathrm{m}$ pores were reduced to 1-3 $\mu \mathrm{m}$.
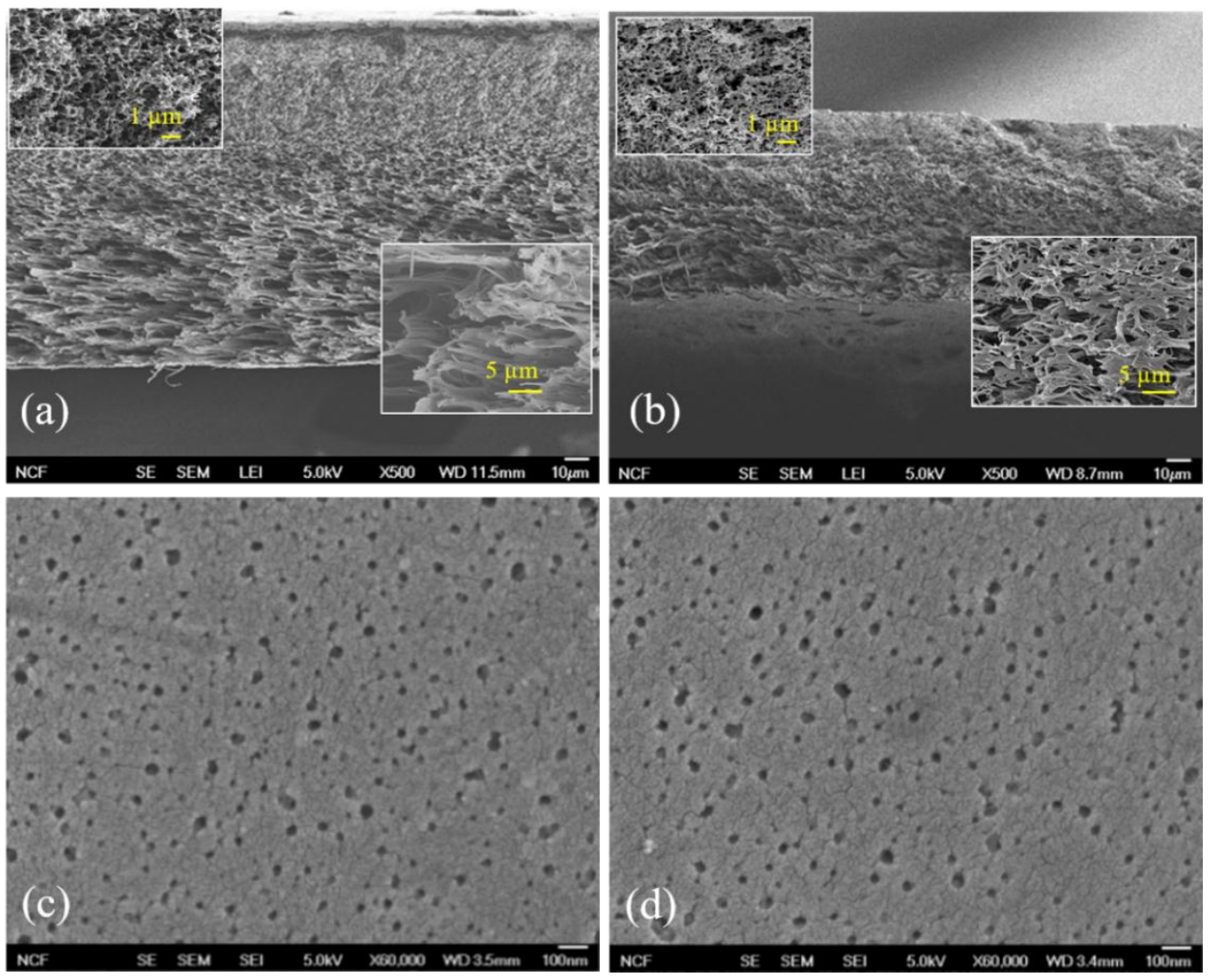

Figure 4. SEM images of the cross-section and surface of the PES-A14 before (a and c) and after (b and d) being compressed at $180{ }^{\circ} \mathrm{C}$ and $4 \mathrm{MPa}$ for $3 \mathrm{~min}$. The insets in (a) and (b) are magnified images of the top and bottom regions of the support layer.

In contrast, SEM measurements (Fig. 4c and 4d) revealed that the top (feed) surface pore size of the PES-A14 remained essentially unchanged after imprinting, which is consistent with the assumption that the Poisson's ratio of the membrane was effectively zero during compression such that no noticeable lateral expansion or distortion of the surface pores occurred. In addition, the 
virgin and compressed membranes showed surface coverage of the pores as $5.1 \%$ and $6.1 \%$, respectively, from ImageJ analysis (corresponding binary plot and threshold are provided in the supplementary material, Fig. S4); these values are typical for UF membranes [29]. Note that the surface coverage of the pores is not equivalent to the volumetric porosity within the skin layer, which most likely decreases upon compression.
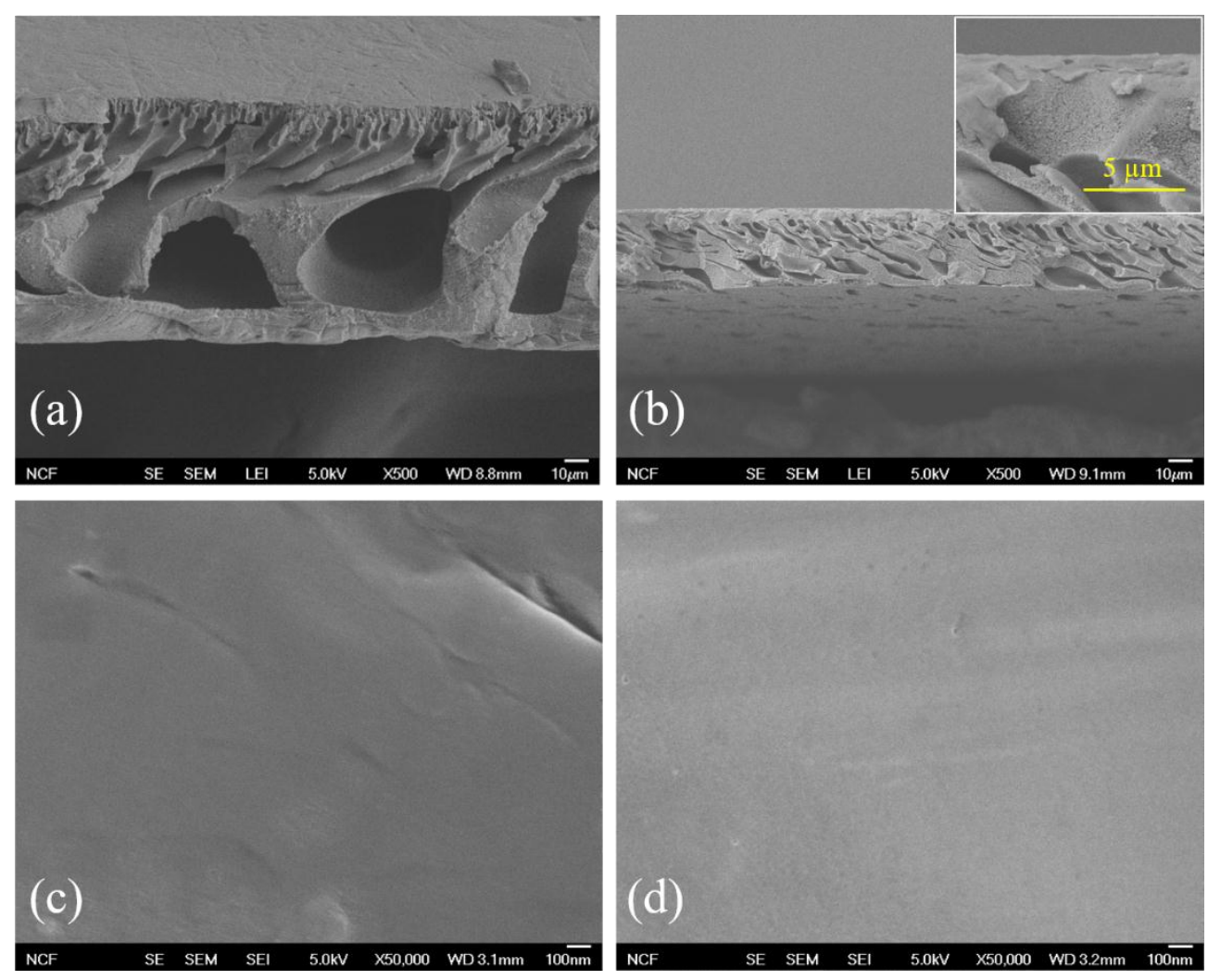

Figure 5. SEM images of the cross-section and surface of the PS membranes before (a and c) and after (b and d) being compressed at $80{ }^{\circ} \mathrm{C}$ and $5.0 \mathrm{MPa}$ for $3 \mathrm{~min}$. The corresponding compressive strain is $49.6 \%$. The insets in (a) and (b) are magnified images of the top and bottom regions of the support layer.

PS membrane displayed even more asymmetric pore structures, containing macrovoids on the order of tens of microns in size (Fig. 5a). After compression, these macrovoids were severely compressed (Fig. 5b), compared with the top selective layer. This is due to the higher porosity (lower yield strength according to Eqn. (2)) in the macrovoids-containing layer. These 
observations are consistent with previously studied UF membranes with similar structures [14, 15]. However, the surface pores of the PS membranes could not be resolved with the FE-SEM (Fig. 5c and 5d), suggesting that the pore size is in the nm-range, which is comparable with the fine morphology of the gold film deposited on these membranes.

Compared with the UF membranes discussed above, both PVDF MF membranes displayed more symmetric pore structures (Fig. 6a for PVDF-VVPP and Fig. S4a for PVDF-DVPP). Correspondingly, the pore structure of the compressed membranes appeared similar across the thickness of the membranes (Fig. 6b and Fig. S4b). The relatively rough and fibrous surfaces of both membranes were flattened after the NIL-compression. (Fig. 6c-6d and Fig. S4).
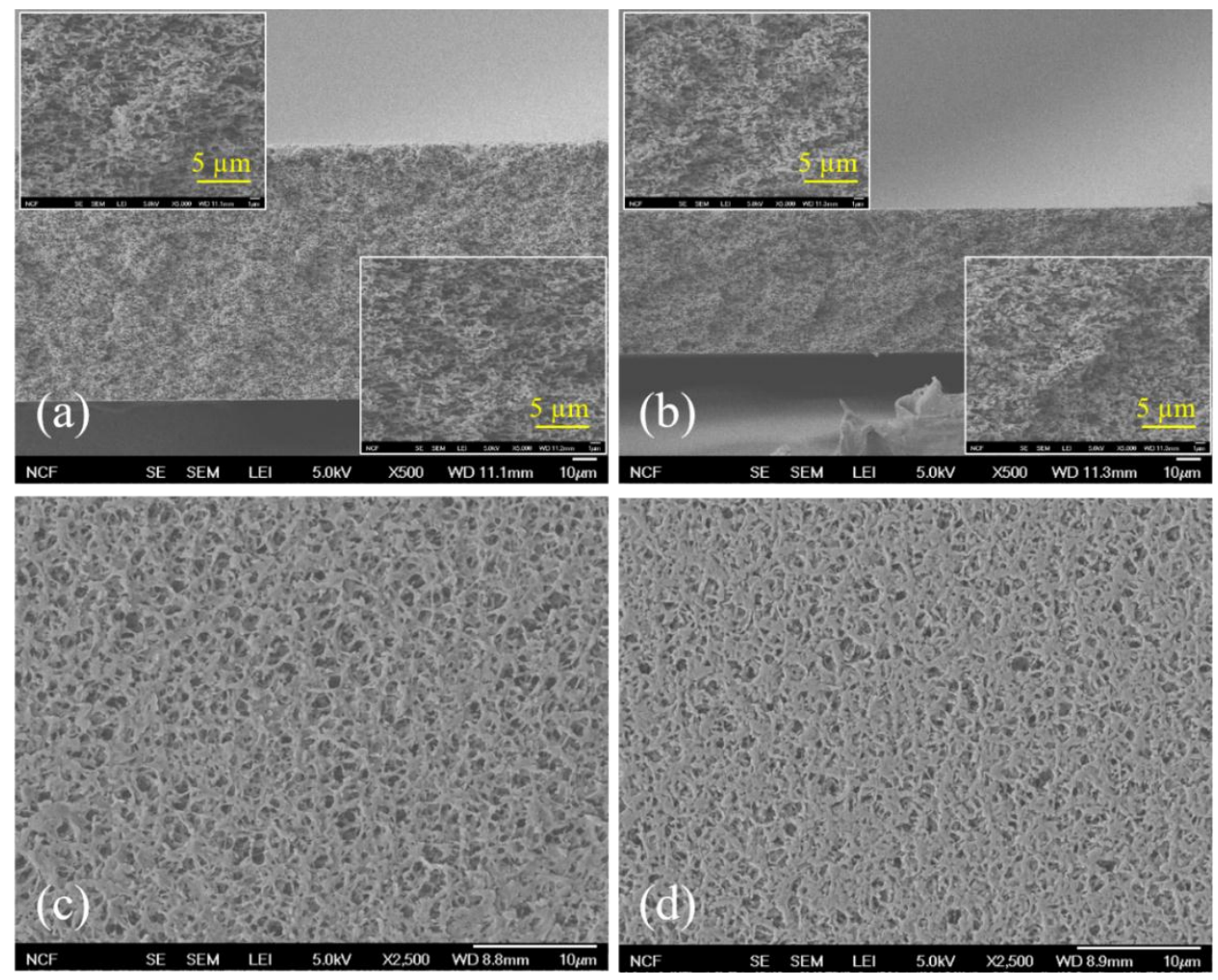

Figure 6. SEM images of the cross-section and surface of the PVDF-VVPP before (a and c) and after ( $\mathrm{b}$ and d) being compressed at $90{ }^{\circ} \mathrm{C}$ and $5.5 \mathrm{MPa}$ for $3 \mathrm{~min}$. The corresponding compressive strain is $\sim 38.0 \%$. The insets in (a) and (b) are magnified images of the top and bottom regions of the support layer. 
Figure 7a summarizes the DI water flux of the compressed membranes as a function of the compressive strain resulting from NIL processing. Different strain levels were achieved by adjusting the temperature and pressure of NIL process. Also note that the flux was normalized by the value obtained for the same membrane sample before compression. The results indicate that the flux of the compressed membranes decreased monotonically with an increase of compressive strain. Qualitatively, it is most likely that mechanical compression alters the major characteristics of a porous structure: pore size and pore-size distribution, porosity, and tortuosity, which collectively contribute to the reduction of DI water flux under the same transmembrane pressure. Interestingly, the dependence of normalized flux on the compressive strain was similar for the four types of membranes examined. This is a surprising result given the significantly different pore characteristics of the UF and MF membranes. From the SEM images, all four membranes showed different degrees of asymmetry and rather different pore sizes (Fig. 4-6, Fig. S4). Despite these clear differences, the flux-deformation relationships were quite similar.

(a)

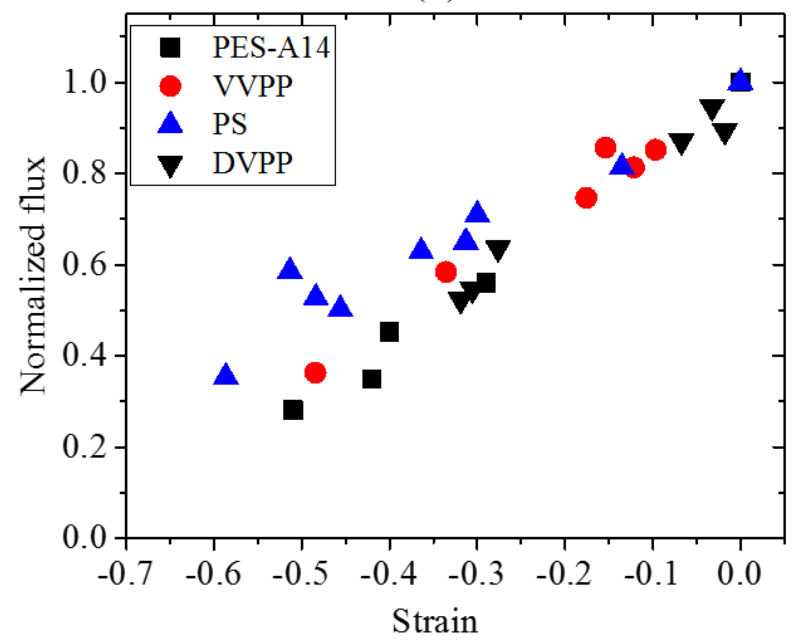

(b)

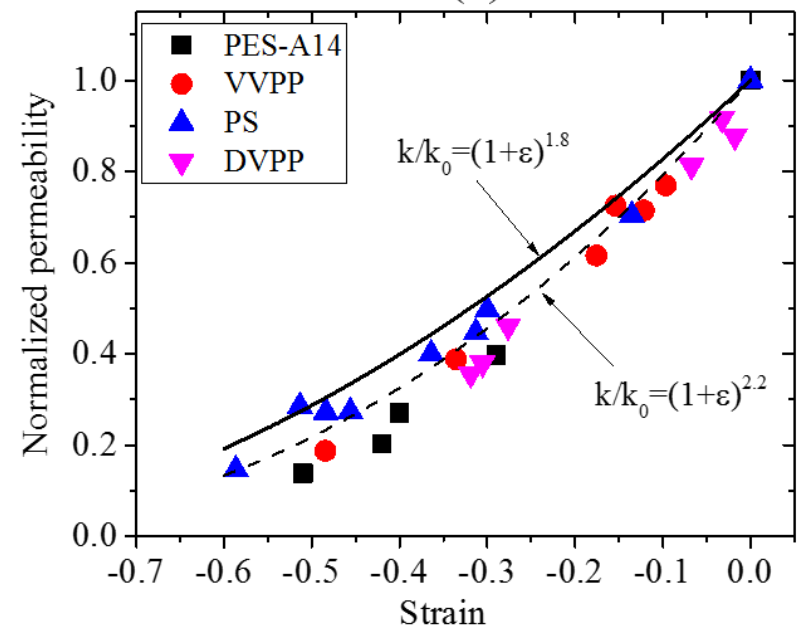

Figure 7. (a) DI water flux and (b) permeability of the compressed membranes, normalized by the values of corresponding virgin membranes, as a function of compressive strain for all four membranes as labelled. Solid and dashed lines in (b) represent predictions from Eq. (5) with $n$ $=1.8$ and 2.2 , respectively. 
As noted previously, we are unaware of any published quantitative relationships between permeation and deformation for separation membranes. On the other hand, it is known that the intrinsic permeability $(k)$ of a high-porosity, open-cell polymer foam depends upon compressive strain, [30]

$$
\frac{k_{\varepsilon}}{k_{0}}=(1+\varepsilon)^{n}
$$

where $k_{\varepsilon}$ and $k_{0}$ are the permeability of the compressed and virgin membranes, respectively. Experimentally, it has been reported that Eq. (5) with exponent $n=1.8$ can describe the data by Gent et al. [31] and recently Dawson et al. [30], while $n=2.2$ can better describe data by Hilyard et al. [32]. Figure $7 \mathrm{~b}$ plots the normalized permeability $\left(k_{\varepsilon} / k_{0}\right)$ for all four membranes in this study based upon the data presented in Fig. 7a. Note that since the transmembrane pressure was identical for the virgin and compressed membranes (see the supplementary material for the detailed derivation),

$$
\frac{k_{\varepsilon}}{k_{0}}=\frac{J_{\varepsilon}}{J_{0}}(1+\varepsilon)
$$

where $J_{0}$ and $J_{\varepsilon}$ are the flux of the virgin and compressed membrane with a strain of $\varepsilon$, correspondingly. Interestingly, the experimental data for all four membranes can be reasonably well described by Eq. (5) with $n=2.2$ or with $n=1.8$ as the upper bound. Such good agreement is unexpected given the aforementioned differences in structure among the four membranes.

Dawson et al developed a model to describe the permeation-deformation behavior of lowdensity open-cell foams [30]. At small strain levels (below yielding), the behavior of the foam follows Eq. (5) with $n=1$. At strain levels above the elastic limit, the foams deform plastically with non-uniform strain, i.e. some regions evidence only small elastic strains while others 
plastically deform with much larger strains. In addition, the portion of the foam that undergoes plastic deformation increases with the increasing overall strain. Therefore, the overall deformation and permeability of the entire foam is a combination of the two regions, each with its own strain and permeability. The model based on such a non-uniform porous structure (Eq. (15) in ref. [30]) can be well approximated by Eq. (5), which was used to describe experimental results from the four membranes with $n=1.8$ or 2.2. We speculate that the observed open-cell foam behavior of the UF and MF membranes used in the subject experiments is due to similar non-uniform deformation and corresponding permeation characteristics. This hypothesis is consistent with the observation that after compression, the bottom of the support layer of the PES-A14 membrane experienced more compression than the top layer (Fig. 4a and 4b) as inferred from the overall reduction in pore size. It is worth noting that the permeation-deformation behavior will likely deviate from that predicted by Eq. (5) if the permeation of the membrane cannot be approximated as an open-cell foam, as is likely for the case of thin film composite membranes. However, the results suggest that the permeation-deformation response characteristic of the porous supports in these membranes may also be described by Eq. (5).

\section{Conclusion}

Porous polymeric membranes are prone to deformation due to the inherent viscoelasticity of the polymer and the high level of porosity. In combination, these characteristics give rise to the well-known occurrence of membrane compaction (creep) during pressure-driven separation. Membrane compaction is generally associated with a decline in performance due to a decrease in permeation over time. On the other hand, porosity-induced plasticity can be utilized to advantage for new applications such as surface patterning. In each case an obstacle to further insight has been the lack of a quantitative relationship between membrane permeability and strain. In this 
work several MF and UF membranes with different pore structure and chemistry were deformed under controlled conditions of temperature, pressure and duration using NIL processing to obtain different levels of plastic deformation. The results showed that despite the differences in pore structure and chemistry, the response of all four membranes was well-described by a permeationdeformation relationship developed for open-cell foams.

\section{Acknowledgement}

The authors gratefully acknowledge funding support from the National Science Foundation under Grant No. CBET-1264276, and IIP- 1432952. The authors also gratefully acknowledge the National Science Foundation (NSF) Industry/University Cooperative Research Center for Membrane Science, Engineering and Technology (MAST) at the University of Colorado at Boulder (CU-B) for support of this research via NSF Award IIP 1624602. We acknowledge the support from the Brazil Scientific Mobility Program / CAPES, Coordination for the Improvement of Higher Education Personnel - Brazil for the support of Gabriel Pichorim. 


\section{References}

[1] G.M. Geise, H.S. Lee, D.J. Miller, B.D. Freeman, J.E. McGrath, D.R. Paul, Water purification by membranes: The role of polymer science, J. Polym. Sci. Part B-Polym. Phys., 48 (2010) $1685-1718$.

[2] Q. She, D. Hou, J. Liu, K.H. Tan, C.Y. Tang, Effect of feed spacer induced membrane deformation on the performance of pressure retarded osmosis (PRO): Implications for PRO process operation, J. Membr. Sci., 445 (2013) 170-182.

[3] D. Paul, O. Ebra - Lima, Pressure - induced diffusion of organic liquids through highly swollen polymer membranes, J. Appl. Polym. Sci., 14 (1970) 2201-2224.

[4] Y.A. Hussain, M.H. Al-Saleh, A viscoelastic-based model for TFC membranes flux reduction during compaction, Desalination, 344 (2014) 362-370.

[5] K.W. Lawson, M.S. Hall, D.R. Lloyd, Compaction of microporous membranes used in membrane distillation. I. Effect on gas permeability, J. Membr. Sci., 101 (1995) 99-108.

[6] H. Ohya, Expression method of compaction effects on reverse-osmosis membranes at highpressure operation, Desalination, 26 (1978) 163-174.

[7] L. Brinkert, N. Abidine, P. Aptel, On the relation between compaction and mechanical properties for ultrafiltration hollow fibers, J. Membr. Sci., 77 (1993) 123-131.

[8] P.F. Fuls, M.P. Dell, I.A. Pearson, Non-linear flow through compressible membranes and its relation to osmotic pressure, J. Membr. Sci., 66 (1992) 37-43.

[9] Y. Mashiko, Y. Kurokawa, S. Saito, Initial flux decline of the cellulose-acetate butylate membranes with time under RO performance, Desalination, 48 (1983) 147-160. 
[10] R.A. Peterson, A.R. Greenberg, L.J. Bond, W.B. Krantz, Use of ultrasonic TDR for real-time noninvasive measurement of compressive strain during membrane compaction, Desalination, $116(1998)$ 115-122.

[11] I.H. Huisman, B. Dutre, K.M. Persson, G. Tragardh, Water permeability in ultrafiltration and microfiltration: Viscous and electroviscous effects, Desalination, 113 (1997) 95-103.

[12] K.M. Persson, V. Gekas, G. Trägårdh, Study of membrane compaction and its influence on ultrafiltration water permeability, J. Membr. Sci., 100 (1995) 155-162.

[13] R.D. Deanin, B. Baum, S.A. Margosiak, W.H. Holley Jr, Effect of fillers on wet compressive creep of cellulose acetate, Industrial \& Engineering Chemistry Product Research and Development, 9 (1970) 172-175.

[14] K.W. Lawsen, M.S. Hall, D.R. Lloyd, Compaction of microporous membranes used in membrane distillation. I. Effect on gas permeability, J. Membr. Sci., 101 (1995) 99108.

[14] S.H. Maruf, L. Wang, A.R. Greenberg, J. Pellegrino, Y. Ding, Use of nanoimprinted surface patterns to mitigate colloidal deposition on ultrafiltration membranes, J. Membr. Sci., 428 (2013) 598-607.

[15] S.H. Maruf, Z.W. Li, J.A. Yoshimura, J.L. Xiao, A.R. Greenberg, Y.F. Ding, Influence of nanoimprint lithography on membrane structure and performance, Polymer, 69 (2015) 129137.

[16] S.H. Maruf, M. Rickman, L. Wang, J. Mersch IV, A.R. Greenberg, J. Pellegrino, Y. Ding, Influence of sub-micron surface patterns on the deposition of model proteins during active filtration, J. Membr. Sci., 444 (2013) 420-428. 
[17] S.H. Maruf, A.R. Greenberg, J. Pellegrino, Y. Ding, Critical flux of surface-patterned ultrafiltration membranes during cross-flow filtration of colloidal particles, J. Membr. Sci., 471 (2014) 65-71.

[18] S.H. Maruf, A.R. Greenberg, Y.F. Ding, Influence of substrate processing and interfacial polymerization conditions on the surface topography and permselective properties of surfacepatterned thin-film composite membranes, J. Membr. Sci., 512 (2016) 50-60.

[19] S.H. Maruf, A.R. Greenberg, J. Pellegrino, Y. Ding, Fabrication and characterization of a surface-patterned thin film composite membrane, J. Membr. Sci., 452 (2014) 11-19.

[20] Y. Ding, S. Maruf, M. Aghajani, A.R. Greenberg, Surface patterning of polymeric membranes and its effect on antifouling characteristics, Separ. Sci. and Technol., in press.

[21] Z. Wang, C. Hansen, Q. Ge, S.H. Maruf, D.U. Ahn, H.J. Qi, Y.F. Ding, Programmable, pattern-memorizing polymer surface, Adv. Mater., 23 (2011) 3669-3674.

[22] L.M. Cox, J.P. Killgore, Z.W. Li, R. Long, A.W. Sanders, J.L. Xiao, Y.F. Ding, Influences of substrate adhesion and particle size on the shape memory effect of polystyrene particles, Langmuir, 32 (2016) 3691-3698.

[23] L.M. Cox, J.P. Killgore, Z.W. Li, Z. Zhang, D.C. Hurley, J.L. Xiao, Y.F. Ding, Morphing metal-polymer Janus particles, Adv. Mater., 26 (2014) 899-904.

[24] B. Nandan, L. Kandpal, G. Mathur, Glass transition behaviour of poly (ether ether ketone)/poly (aryl ether sulphone) blends: dynamic mechanical and dielectric relaxation studies, Polymer, 44 (2003) 1267-1279.

[25] S. Hellinckx, J.-C. Bauwens, The yield behavior of PVDF and the deformation process at high temperature, Colloid \& Polym. Sci., 273 (1995) 219-226. 
[26] C. Plummer, A. Donald, The ductile-brittle transition in macroscopic tensile tests on polyethersulfone, J. Appl. Polym. Sci., 41 (1990) 1197-1212.

[27] L.J. Gibson, M.F. Ashby, Cellular solids: structure and properties, Cambridge university press, 1999.

[28] N.P. Cheremisinoff, Handbook of polymer science and technology, CRC Press, 1989.

[29] K. Kim, A. Fane, C. Fell, T. Suzuki, M. Dickson, Quantitative microscopic study of surface characteristics of ultrafiltration membranes, J. Membr. Sci., 54 (1990) 89-102.

[30] M. Dawson, J. Germaine, L. Gibson, Permeability of open-cell foams under compressive strain, Int. J. Solids and Struct., 44 (2007) 5133-5145.

[31] A. Gent, K. Rusch, Permeability of open-cell foamed materials, J. Cell. Plast., 2 (1966) 4651.

[32] N. Hilyard, P. Collier, A structural model for air flow in flexible PUR foams, Cell. Polym., 6 (1987) 9-26. 


\section{Graphic Abstract}
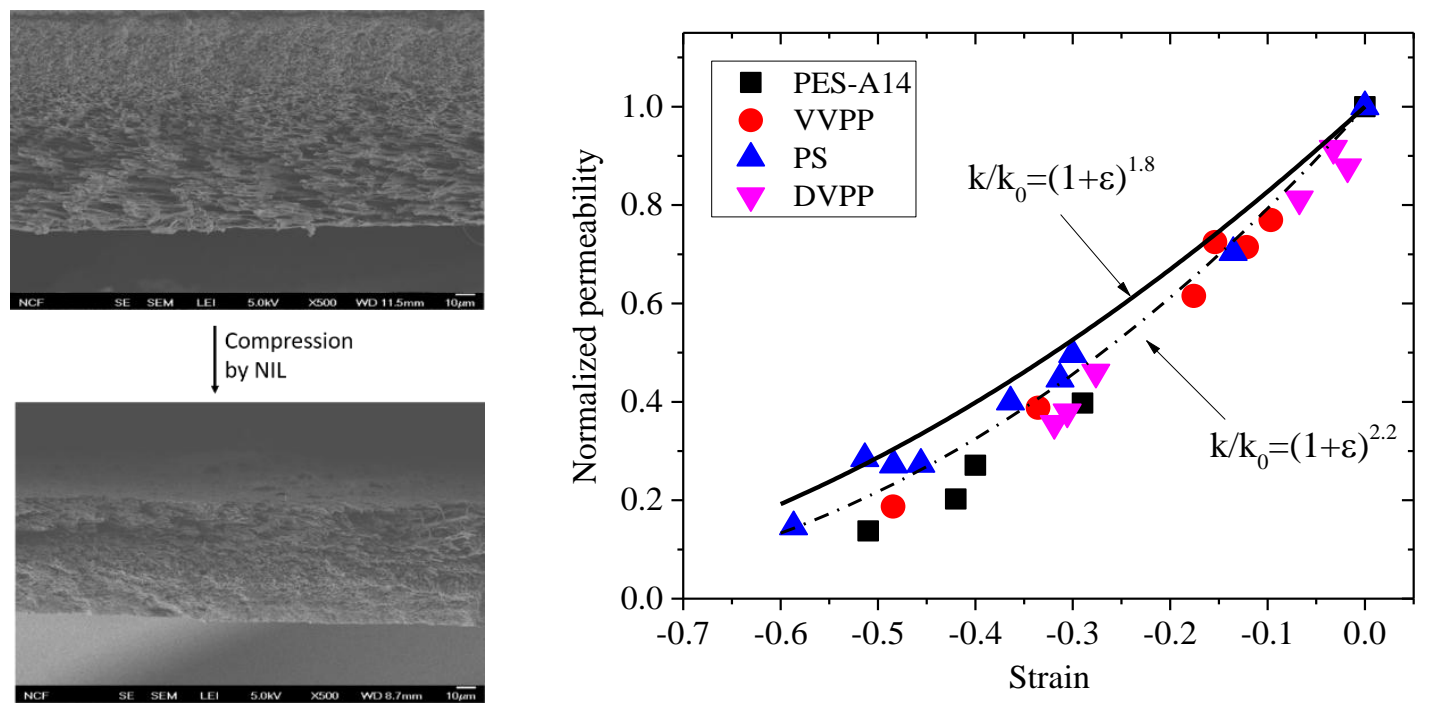

Left: SEM images showing thickness reduction of a UF membrane before (top) and after (bottom) the compressive deformation exerted by NIL. Right: Normalized membrane permeability as a function of compressive strain for four different membranes measured. 